\title{
Content based Image Retrieval (CBIR) System using Threshold based Color Layout Descriptor (CLD) and Edge Histogram Descriptor (EHD)
}

\author{
Pradnya A. Vikhar \\ Research Scholar, \\ SSGB Amravati University, Amravati
}

\begin{abstract}
The accuracy of any Content Based Image Retrieval (CBIR) system is depend on the accuracy of features extracted from the images. Therefore feature extraction is the first and most important step to develop the CBIR system. Instead of using single feature, most common CBIR system uses combination of color, texture and/or shape to accurately represent an image. MPEG-7 feature extraction provides standards set of descriptors to represent the images and are widely used in many applications.

In this paper the concept and area of content based image retrieval system is introduced first. Further it covers the overview of basic MPEG-7 visual feature descriptor; CLD (Color Layout Descriptor) and edge histogram descriptor (EHD) to extract the contents from images. To reduce the overall searching process the advancement in CLD and EHD is suggested by introducing the concept of thresholding.
\end{abstract}

\section{General Terms}

Image Retrieval, MPEG-7 feature Extraction Descriptors.

\section{Keywords}

CBIR, Feature extraction, MPEG-7, Color Layout Descriptor (CLD), Edge Histogram Descriptor (EDH)

\section{INTRODUCTION}

Due to rapid enhancement in digital technology, huge amount of digital information in the form of images and videos is available in recent years. Also gigabytes of new digital information is generated and transmitted everyday [1][2]. Today's technology demands the use of this digital information in form of image in various domains including bioinformatics, medicine, entertainment, designing, advertisement and engineering science. All this visual information is useless, if there is no proper mechanism to effectively store and retrieve these images.

In early days, images were represented by text and then textbased concept of traditional database management system was used to search and retrieve images. As the approach is based on text-based search, it is commonly known as Text-based Image Retrieval (TBIR) [3]. The problems in front of TextBased image retrieval (TBIR) system became more severe, as volume of digital images produced increased dramatically in various fields of science and engineering. The other and main difficulty faced by this approach is to describe rich content in image manually. It is very expansive and obviously subjective task. This is driving force behind the emergence of Content Based Image Retrieval (CBIR) system [1][3]. In CBIR system manual representation of images by text- based key words is replaced by, their own visual content, such as color, texture

\author{
P. P. Karde \\ $\mathrm{PhD}$, Head, Department of Computer Engineering, \\ Government Polytechnic, Amravati
}

and shape. Since 1990s, it is an active area of research and development. Exhaustive efforts have been undertaken by many researchers to build efficient and effective CBIR system. Most of the work in this area is focused on feature extraction, similarity matching, indexing (to sort output images based on certain attributes), relevance feedback (take users view to display the result). [1][2]. The study of literature proves that if the number of features are combined the resultant CBIR system gives accurate output. [4]

The purpose of this paper is to focus on feature extraction using Color Layout Descriptor (CLD) and Edge Histogram Descriptor (EHD)[5][6]. CLD and EHD belongs to family of MPEG-7 descriptor standard which includes standardized tools to enable detail description of visual information. To develop more efficient CBIR system, these descriptors are refined and modified using the concept of thresholding. This will reduce the search space which results in efficient CBIR system.

\section{CONTENT BASED IMAGE RETRIEVAL SYSTEM}

Application involving automatic indexing, searching, retrieving and browsing of image databases uses concept of Content based image retrieval (CBIR) systems. It is technique which uses visual content (color, shape and texture) of image to search large image database as per users' interest [1][2]. A typical CBIR system is shown in following figure [2]-

In CBIR system, the input query given by user is in the form of image. It has to search the relevant images similar to input image from image database. For this feature extraction of query image and images present in database is done based on certain visual features. It forms feature space (digital representation of image using visual feature).

Then feature vector of query image is matched with feature vectors of images present in feature database. After similarity matching, most similar images results as an output. Thus in the CBIR system, the input given and output produced are both in form images [3]. 


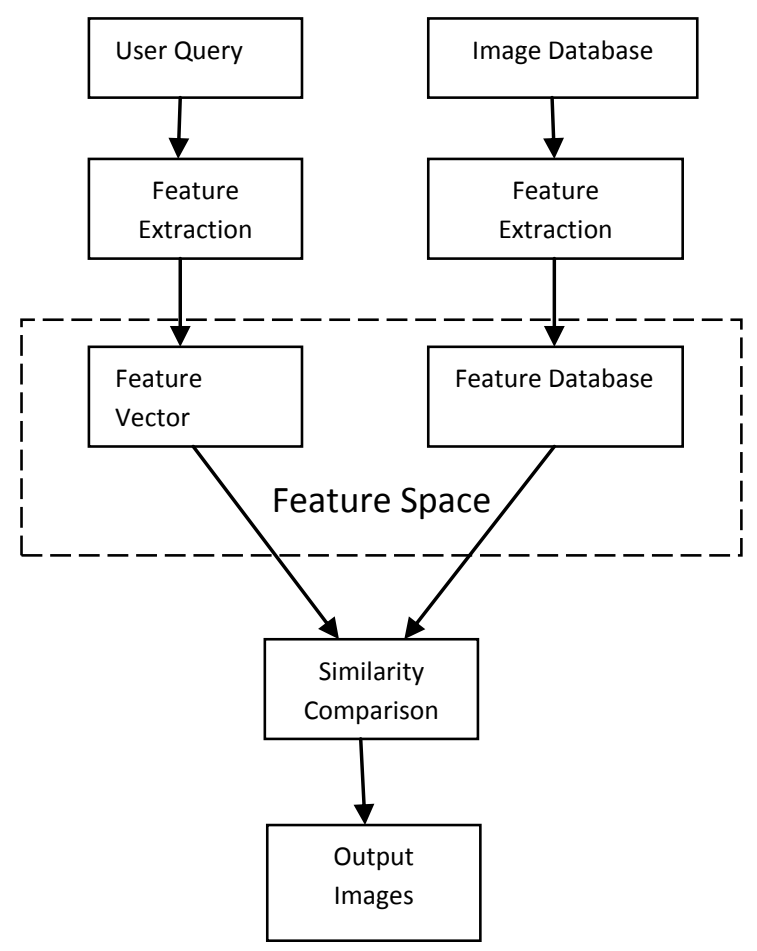

Figure 1: A typical view of CBIR System

\section{COLOR LAYOUT DESCRIPTOR}

In order to describe and interpret visual contents from images, MPEG-7 standard provides different Multimedia Descriptors. These MPEG-7 visual standard descriptors offer interoperability across different multimedia database systems [6]. MPEG-7 provides standardized descriptors for visual, audio and video retrieval. Main visual features descriptors are color descriptors, shape descriptors and texture descriptors.

A color layout descriptor (CLD) is used to extract the spatial distribution of color in an image. The feature extraction process divided into two parts; grid based representative color selection and discrete cosine transform with quantization.

Color is the most important visual content to recognize or identify the image, therefore it is possible to use colors to describe and represent an image. The MPEG-7 standard has tested the most efficient procedure to describe the color and has selected those that have provided more satisfactory results.

The typical steps to extract the features using CLD are as follows-

1. The image is divided into 64 blocks ( 8 rows $\mathrm{X} 8$ columns).

2. A representative color was chosen for each block by averaging the RGB values of all the pixels in that block. This results in three $8 \times 8$ arrays, one for each color component.

3. Each $8 \times 8$ matrix was transformed to the $\mathrm{YCbCr}$ color space.

4. These will be again transformed by $8 \times 8$ DCT (Discrete Cosine Transform) to obtain three $8 \times 8$ DCT matrices of coefficients, one for each $\mathrm{YCbCr}$ component.

5. The CLD descriptor was formed by performing zigzag scanning with these three sets of 64 DCT coefficients.
The purpose of the zigzag scan is to group the low frequency coefficients of the $8 \times 8$ matrix.

\subsection{Procedure to form the threshold based clusters using CLD}

For each image in dataset match ratio is calculated-

$$
\mathrm{MRi}=\int_{\mathrm{i}=1}^{\mathrm{n}} \frac{\text { Rchannel }+ \text { Gchannel }+ \text { Bchannel }}{3} \times \mathrm{CR}
$$

Where: MR- Match ratio of image $i$

Rchannel, Gchannel, Bchannel- Values of R,G,B optioned after applying CLD algorithm

$$
\begin{aligned}
& \mathrm{CR}=(1 / 1000) \text { constant } \\
& \text { use to make truncation }
\end{aligned}
$$

Threshold value is calculated from the input search image

$$
C T h=\frac{\sum V r, V g, V b}{3} \times C R
$$

Where: $\mathrm{Vr}, \mathrm{Vg}, \mathrm{Vb}$ - Value of R,G,B for searching image

$$
\begin{aligned}
& \mathrm{CTh}=\text { Threshold value for CLD } \\
& \mathrm{CR}=\text { Constant divider }
\end{aligned}
$$

\section{EDGE ORIENTATION HISTOGRAM}

To represent the image content more accurately the methodology suggested here uses, popularly used texture descriptor: Edge Histogram Descriptor (EHD) along with Color Layout Descriptor (CLD).

The EHD [4][6][7] is used to characterizes edges in form of spatial distribution in an image. The process of feature extraction using EHD consists of following steps:

1. The sub-image is defined by dividing the image space into $4 \times 4$ non-overlapping blocks. Thus, the image partition always yields 16 equal-sized subimages regardless of the size of the original image.

2. To characterize the sub-image, then generate a histogram of edge distribution for each sub-image.

3. Edges in the sub-images are categorized into 5 types: vertical, horizontal, 45-degree diagonal, 135degree diagonal and non-directional edges.

4. Thus, the histogram for each sub-image represents the relative frequency of occurrence of the 5 types of edges in the corresponding sub-image.

5. As a result, each local histogram contains 5 bins. Each bin corresponds to one of 5 edge types. Since there are 16 sub-images in the image, a total of $5 \times 16=80$ histogram bins is required.

By applying above steps, the texture features are extracted from the input image and all the images present in image database.

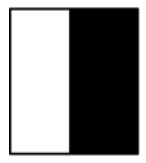

horizontal

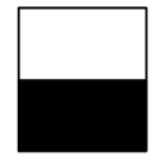

vertical

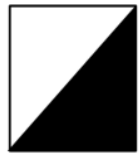

45 degree

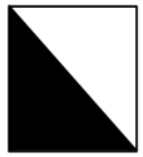

135 degree non-directional 

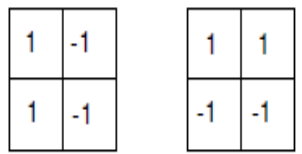

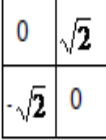

Figure 2. Five types of edges and corresponding filters [7]

\subsection{Procedure to form the threshold based clusters using EHD}

For each image in dataset match ratio is calculated-

$$
\mathrm{MWi}=\int_{\mathrm{i}=1}^{\mathrm{n}} \frac{\mathrm{Ev}+\mathrm{Eh}+\mathrm{Ed} 1+\mathrm{Ed} 2 \_\mathrm{En}}{5} \times \mathrm{CR}
$$

Where: MWi- Match weight of image i

Ev,Eh,Ed1,Ed2,En- Edge values obtained by calculated values of 5bins i.e vertical edge, horizontal edge, 450 diagonal, 135o diagonal and non-direction edge respectively

$\mathrm{CR}=(1 / 1000)$ constant use to make truncation

Threshold value is calculated from the input search image

$$
E T h=\frac{\sum V v, V h, V d 1, V d 2, V n}{5} \times C R
$$

Where: Vv, Vh,Vd1,Vd2,Vn- Value of 5 bins obtained after applying EHD on searching image

$\mathrm{ETh}=$ Threshold value for EHD

$\mathrm{CR}=$ Constant divider

\section{METHODOLOGY}

In this paper, Threshold based Color Layout Descriptor (CLD) and Edge Histogram Descriptor (EHD) of MPEG-7 is used to develop the Content Based Image Retrieval system. By using threshold based CLD and EHD the color and texture features of image is extracted [8][9]. The true positive and true negative images based on the threshold value are calculated and the result is displayed.

The major steps involved in the proposed methodology are as follows:

Step 1: All the images initially are stored in a directory, called image database. Directory of image database is selected first.

Step 2: Through interface, user select Query image as an input.

Step 3: Using the CLD the color features of input image is extracted, the threshold value is computed.

Step 4: By executing threshold based the Edge Histogram Descriptor (EDH), the threshold value of 5 bins received by applying EHD of image is calculated.

Step 5: Similarly thresholds of all images present in image database is calculated.

Step 6: Based on the threshold value of input image, true positive and true negative images are calculated.

Step 7: True positive images searched by applying threshold based CLD and EHD are displayed as the result of retrieval process.

\section{RESULT AND DISCUSSION}

The approach discussed here is implemented in JAVA. The interface is developed which select image database and generate (load) feature database. Further user select query image whose feature vector is compared with vectors in feature database and output is displayed. The system developed is tested on standard WANG dataset containing 1000 images.

Step 1: User has to first select the input image from the specified directory. This Input the query image is then process to obtain color and texture features of an image.

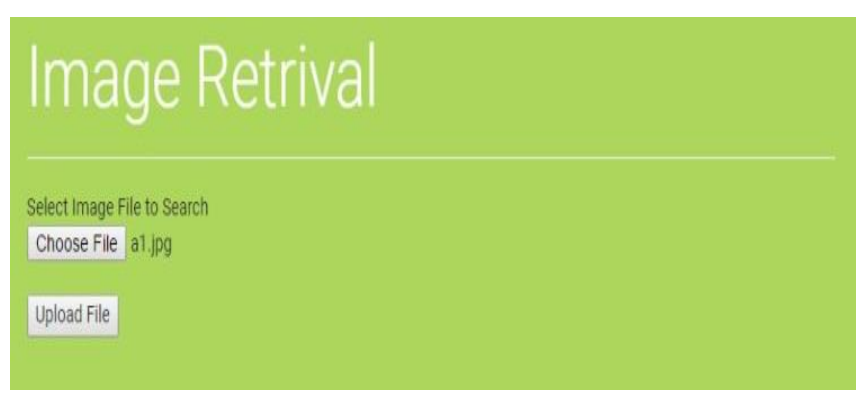

Figure 3. Input Image

Step 2: The input image is then portioned using CLD as shown in following figure. Its Color values are extracted.

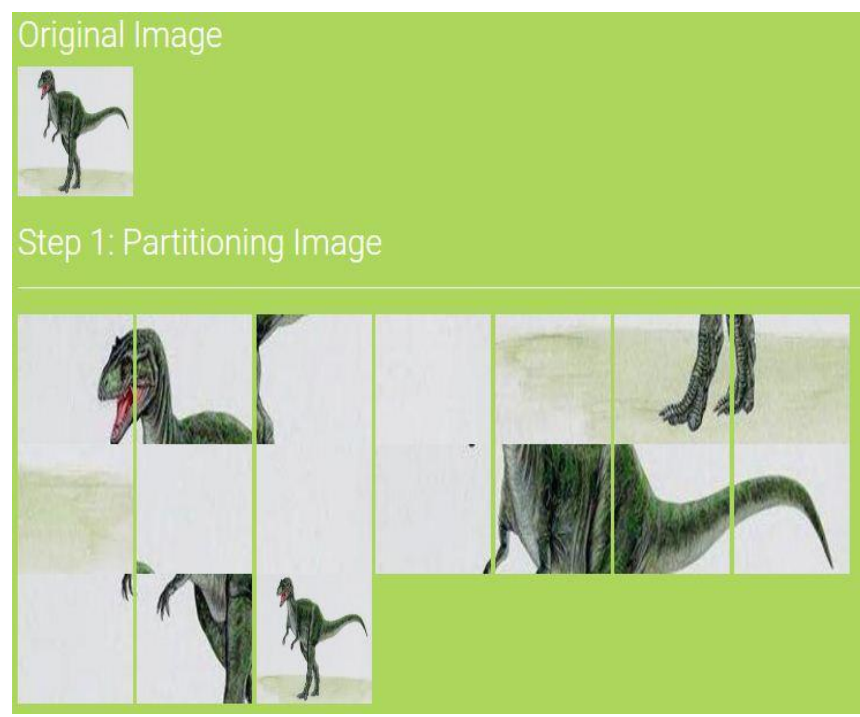

Figure 4. Image partitioning after applying CLD

Step 3: Values of Rchannel, Gchannel and Bchannel after applying CLD are as shown in figure 5. 


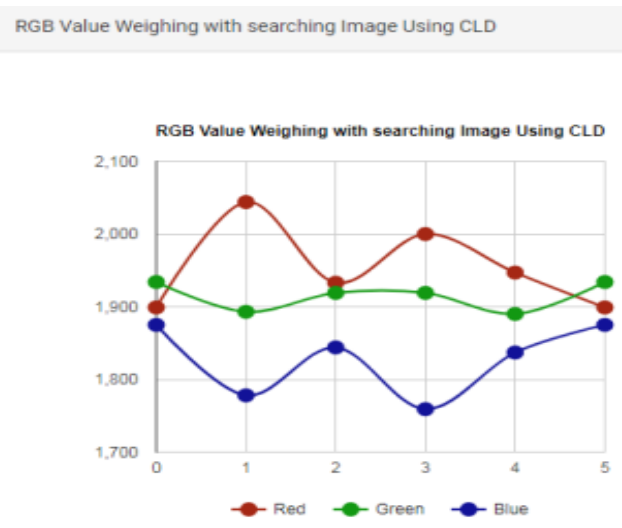

Figure 5. Color Values of input image and other selected images in database

Step 4: By comparing threshold based CLD values of input image and other images present in the image database the search result Threshold based CLD is displayed.

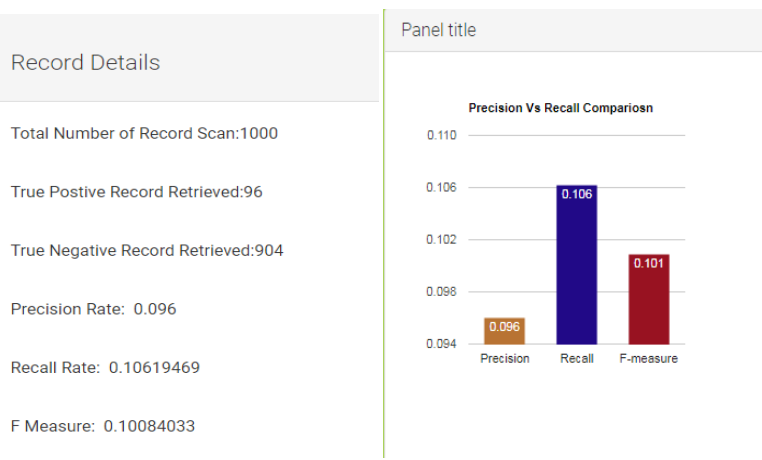

Figure 6. Result of Threshold based CLD

Step 5: Similarly the Threshold values of input image and all images in database are calculated using threshold based EHD

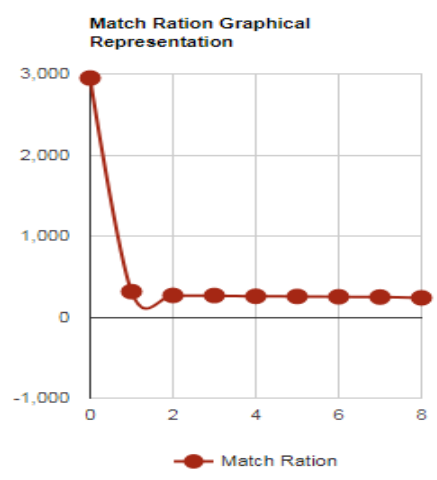

Figure 7. Weight calculated using Threshold based CLD

Step 6: Based on the weight calculated using threshold based EHD, the true positive set of images is displayed as an output.

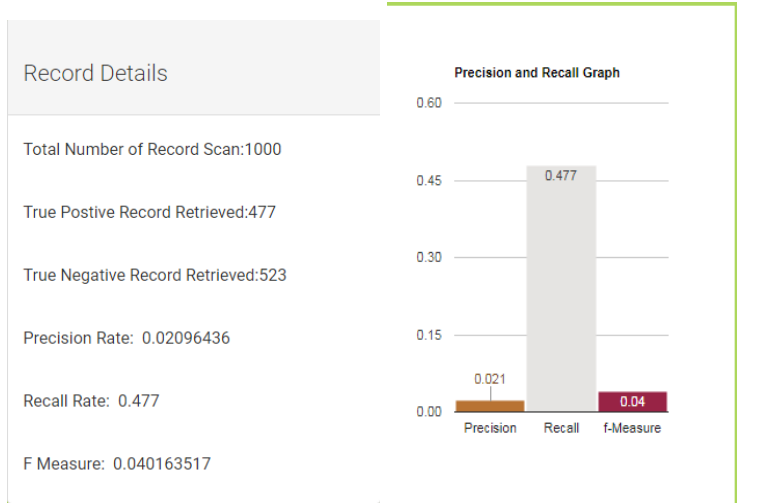

Figure 8. Result Threshold based EHD

Step: 7 The WANG dataset used for experimental purpose contains 1000 images of 10 different categories. For the comparison between Threshold based CLD \& EHD, the sample image from each category is selected and average of precision, recall and fmeasure are calculated are shown in figure 9 and 10.

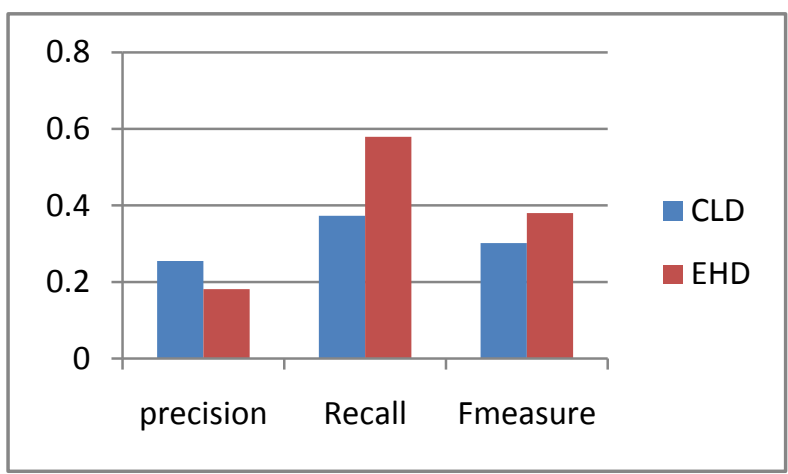

Figure 9. Result of Threshold based CLD and EHD

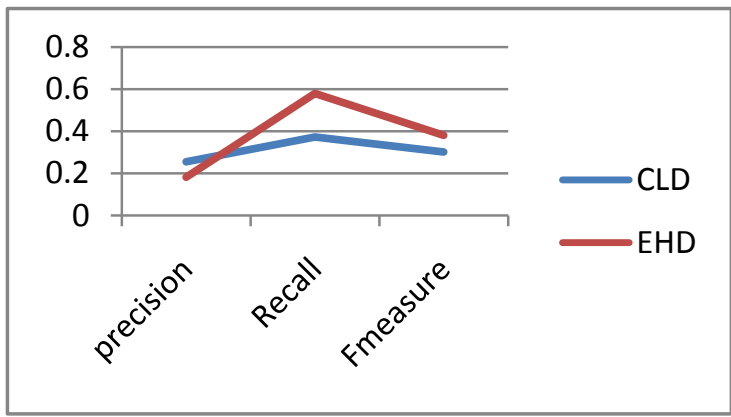

Figure 10. Result of Threshold based CLD and EHD

\section{CONCLUSION}

Image feature extraction is most important step in developing any CBIR system. The accuracy of result and efficiency is also based on the feature extracted technique used for extraction of features. Combination of more than one feature is obviously represent the image more accurately. The methodology presented here uses MPEG-7 Color Layout Descriptor (CLD) and Edge Histogram Descriptor (EHD), as main feature extraction technique. These techniques are further improved using the concept of thresholding to decrease the search space. Reduction in search space is obviously reduces the searching time. The result of system developed using the approach is effective to represent any image contents and reduces the search space to generate the fast output. 


\section{REFERENCES}

[1] Suman Lata, Parul Preet Singh, "A Review on Content Based Image Retrieval System", International Journal of Advanced Research in Computer Science and Software Engineering, Volume 4, Issue 5, PP 561-566, May 2014

[2] Shereena V.B, Julie M. David, "Content Based Image Retrieval: A Review", Computer Science \& Information Technology, Computer Science Conference Proceedings (CSCP), pp. 65-77, 2014

[3] Vikhar Pradnya A. "Content-Based Image Retrieval (CBIR): State-of-the-Art and Future Scope for Research", IUP Journal of Information Technology, Vol. 6,Issue 2, pp. 64-84, June 2010

[4] Shiv Raj Singh, Dr. Shruti Kohli”, Enhanced CBIR using Color Moments, HSV Histogram, Color Auto Correlogram, and Gabor Texture", International Journal of Computer Systems (ISSN: 2394-1065), Volume 02Issue 05, pp 161-165, May, 2015

[5] Jun Zhang, Lei Ye, and Jianhua Ma, "MPEG-7 Visual Descriptors and Discriminant Analysis", The Handbook of MPEG Applications: Standards in Practice, John Wiley \& Sons Ltd, 2011

[6] Laila Shoukry, Sebastian Klenk, Gunther Heidemann, "MPEG-7 Feature Visualization for CBIR Systems", in proceedings of International Conference on Computer Theory and Applications (ICCTA 2010), 2010

[7] Pradnya Vikhar, Pravin Karde, "Improved CBIR System using Edge Histogram Descriptor (EHD) and Support Vector Machine (SVM)", IEEE International Conference on ICT in Business Industry \& Government (ICTBIG), April 2017.

[8] M H Saad, H I Saleh, H Konbor, M Ashour, "Image Retrieval based on Integration between $\mathrm{YCbCr}$ Color Histogram and Shape Feature", IEEE Computer Engineering Conference (ICENCO), 2011, vol., no., pp.97-102, 27-28, 2012

[9] Ching-Hung Sua, Mohd Helmy Abd Wahab and TsaiMing Hsieh, "Image Retrieval based on color and texture features", IEEE 9th International Conference on Fuzzy Systems and Knowledge Discovery (FSKD 2012), PP 1816-1819, 2012.

[10] 